\section{Annual PSA screening might reduce mortality from recurrent prostate cancer}

Annual screening for PSA levels in men who have undergone radical prostatectomy for clinically localized prostate cancer might reduce prostate-cancer-specific mortality, according to a new study by Efstathiou and colleagues.

Preoperative and follow-up data were collected from a total of 1,452 men who had experienced biochemical recurrence after radical prostatectomy. These patients were either taking part in a screening study $(n=841)$ or were attending a community-based practice $(n=611)$. The authors found that men who had participated in the screening study had significantly lower PSA levels at diagnosis $(5.1 \mathrm{ng} / \mathrm{ml}$ versus $9.5 \mathrm{ng} / \mathrm{ml}, P<0.0001)$, were less likely to have Gleason score 7-10 prostate cancer (25.1\% versus $42.1 \%, P<0.0001$ ), and were more likely to have low-risk disease $(64.5 \%$ versus $23.8 \%$, $P<0.0001)$ than those who were attending the community-based practice. The screeningstudy cohort also contained few patients with PSA doubling times of $<12$ months, and a high proportion of patients with a PSA doubling time of $\geq 12$ months. Prostate-cancer-specific mortality after biochemical recurrence was significantly lower in the screening-study cohort than in the community-based cohort (10-year mortality $3.6 \%, 95 \% \mathrm{Cl} \mathrm{1.3-5.8} \mathrm{versus} 11.3 \%$, 95\% Cl 5.9-17.4; $P=0.0002$ ).

The authors conclude that men diagnosed with prostate cancer recurrence as a result of annual PSA screening are more likely to experience an indolent course of disease, and less likely to die of prostate cancer after biochemical failure, than those who are managed in community-based practices.

Original article Efstathiou JA et al. (2006) Prostate-specific antigen-based serial screening may decrease prostate cancer-specific mortality. Urology 68: 342-347

\section{Erectile dysfunction - an early marker of diabetes mellitus?}

Erectile dysfunction and diabetes mellitus share a number of risk factors, and often coexist. As erectile dysfunction is more evident to patients than diabetes mellitus, it has been suggested that erectile dysfunction could be used as an early indicator of diabetes mellitus.
Sun and colleagues conducted a retrospective study into the prevalence of diabetes mellitus in men aged $\geq 18$ years, with $(n=285,436)$ and without $(n=1,584,230)$ erectile dysfunction. Patient data were obtained from a large, US, managed-care claims database. The authors found that the prevalence of diabetes mellitus was almost three times higher in men with erectile dysfunction than in men without erectile dysfunction $(20.0 \%$ versus $7.5 \%$, respectively). After adjustment for age, geographical region and concurrent diseases, this association between diabetes mellitus and erectile dysfunction remained significant $(P<0.0001)$.

Compared with men without erectile dysfunction, men with erectile dysfunction had an overall odds ratio of 1.60 for diabetes mellitus. Age had a nonlinear effect that might limit the usefulness of erectile dysfunction as a marker for diabetes mellitus in older men, however: the peak odds ratio of 2.94 occurred in men aged $26-35$ years, whereas men aged $\geq 66$ years had an odds ratio approaching 1 .

These data confirm that men with erectile dysfunction are more likely to have diabetes mellitus than men without erectile dysfunction. The authors suggest that erectile dysfunction could be a useful early marker of diabetes mellitus in men aged $\leq 45$ years, but this marker is not useful in men aged $\geq 66$ years.

Original article Sun P et al. (2006) Erectile dysfunction-an observable marker of diabetes mellitus? A large national epidemiological study. J Urol 176: 1081-1085

\section{SUl at first pregnancy increases the risk of long-term symptoms}

A study of women who experienced stress urinary incontinence (SUI) during their first pregnancy or shortly after delivery has reported that these women have an increased risk of longterm symptoms, compared with women who did not experience SUI associated with their first pregnancy or delivery.

This longitudinal, cohort study included 241 women. At 12 years after first delivery, the overall prevalence and incidence of SUI were $42 \%$ and $30 \%$, respectively, but the prevalence of SUI was markedly elevated in women who experienced onset of symptoms during their first pregnancy $(56 \%)$ or during their first postpartum period $(78 \%)$, compared with the prevalence in women without onset of symptoms at 